International Journal of Engineering \& Technology, $7(2.12)(2018) 228-230$
SPC

\title{
Fostering a high efficient waveguide diplexer to improve 5G network frequency spectrum for IoT devices in cloud
}

\author{
Gautami Alagarsamy ${ }^{1}$ *, Dr.J.Shanthini ${ }^{1}$ \\ ${ }^{1}$ SNS college of Technology, Coimbatore,TamilNadu,India-641035 \\ *Corresponding author E-mail: aa.gautami@gmail.com
}

\begin{abstract}
In recent days Internet of Things and 5G network connection are complement to each other. The 5G networks would surpass 4GLTE, 4G, $3 \mathrm{G}$ and the other networks we used. It has become a boon for the end users and corporate due to its architecture to handle the heavy data traffic of connected smart devices and large amount of smart phone users worldwide. 5G devices should support longer battery and available at low cost and consume less energy. Some smart phones have ability to charge wirelessly through inductive coupling between base and the phone. In advanced options for charging IoT devices Wireless deportation technology is integrated. To curb network congestion in denser areas and to operate at higher data rates in Ka band applications this research paper analyzes the impact of WR-28 Waveguide Diplexer to improve network frequency spectrum around $30 \mathrm{GHz}$ for IoT devices in cloud services. The design simulation and modeling are implemented by using Antenna Magus version 5.5 Software.
\end{abstract}

Keywords: 5 G Mobile Network, IoT Devices; Network Frequency Spectrum; Waveguide Diplexer; S Parameter; Ka Band Applications.

\section{Introduction}

An ideal wireless network is needed to manage all IoT devices in cloud. To design an ideal network an RF engineer should consider the features such as; the data would download at ultra faster rate, connecting devices pervasively anywhere in the cloud. The smart phones should support the features such as no dropped calls, deliver all the messages or opening a webpage at no time. Currently, our devices cannot download the required amount of data from internet at optimum speed due to heavy trafficking and network congestion [1]. Nowadays the wireless networks possess a frequency spectrum of $1 \mathrm{GHz}$ to $3 \mathrm{GHz}$. This problem challenges can be handled by improving the network frequency spectrum [2]. The necessary data rate is provided by the service providers in order to equate the download speeds. 5G has to operate at a frequency above $6 \mathrm{GHz}$. The network has a capacity to operate up to $100 \mathrm{GHz}$.

In denser network, the hardware should maintain extra capacity with wider bandwidths facility. For a mobile communication network the frequency of $30 \mathrm{GHz}$ is mandatory. To facilitate the network during any emergency situations such as tremor or any natural disasters the frequency range of around $100 \mathrm{GHz}$ helps us to sort any problems. This extra complementary frequency range provides the emergency service to remote areas extensively [3]. To satisfy all these demands a diplexer waveguide is needed for wider network for all IoT devices connected ubiquitously.

\section{Working principle}

A diplexer is a physical device used in $5 \mathrm{G}$ mobile systems, which plays an important role in improving network frequency spectrum [5]. The property of diplexer is it can combine or split the signals into two frequency bands at different ranges using a simplified 2D geometry. The diplexer can receive a signal and transmits a signal at lower and higher frequency respectively. The characteristics of diplexer are predicted by S-parameters and electric fields (emw). To foster an efficient network frequency spectrum the WR-28 waveguide diplexer model is used. It is used for Ka-band application [5] [7]. The lower band of $28 \mathrm{GHz}$ (left) and upper band pass of $30.4 \mathrm{GHz}$ (right) are calibrated to operate a diplexer effectively.

\section{Proposed model}

The waveguide model is considered only for dominant TE1 mode. The width of the $2 \mathrm{D}$ waveguide is 0.28 inches. The length of the longer side of a WR-28 waveguide aperture is 0.28 inches. The cutoff frequency of the dominant mode is $21.08 \mathrm{GHz}$.

There are two cavities working as band pass structures between the input and each output port that is connected with irises. Copper is used to model the waveguides, cavities and irises. Impedance Boundary condition with finite conductivity is maintained to evaluate loss at a high frequency range and the inside of the waveguide is filled with air. A port boundary condition is applied with rectangular TE1 mode, at each end of the waveguide. One port is excited to observe the S-parameters. The Table 1 shows the parameters used to design WR-28 Waveguide Diplexer. 


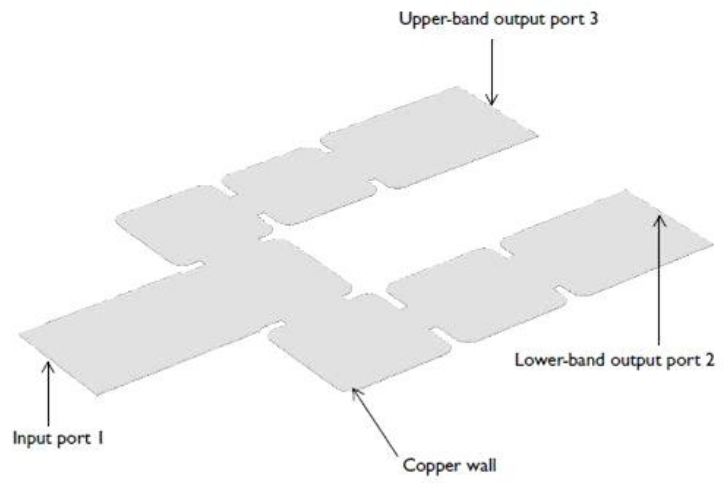

Fig. 1: 2D Layout of the Diplexer Composed of 3-Port Waveguide Structures.

Table 1: Design Parameters of WR-28 Waveguide Diplexer

\begin{tabular}{ll}
\hline Design Parameters & Design Measurements \\
\hline Cut off Frequency & $21.08 \mathrm{GHz}$ \\
Waveguide Width & 0.28 inches \\
Waveguide aperture & 0.28 inches \\
S Parameter & $\mathrm{S} 11$ \\
Lower to upper pass band & $28 \mathrm{GHz}$ to $30.4 \mathrm{GHz}$ \\
Port Dominant mode & Rectangular TE1 mode \\
Insertion Loss & $0.1 \mathrm{~dB}$ \\
\hline
\end{tabular}

\section{Simulation results of WR-28 waveguide di- plexer}

The best method to evaluate a diplexer design is by simulation. The simulation can provide the operating range and proper alignment of diplexer at concurrent iterations. The simulation shows that the input power of $5 \mathrm{G}$ network with distributive nature and independent coupling at each pass band frequency.
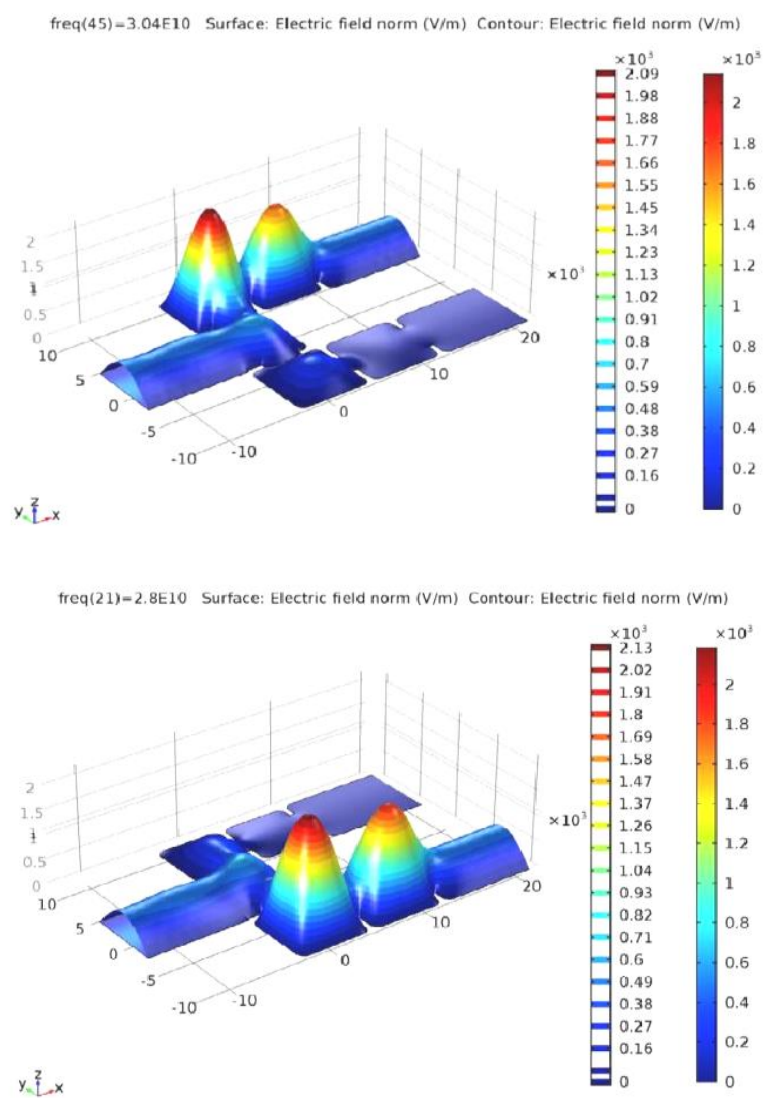

Fig. 2: A Simulated Waveguide Diplexer with Insertion Loss in Each Passband of $0.1 \mathrm{Db}$.

To simulate the lower passband is set around $28 \mathrm{GHz}$ and the upper passband is set around $30.4 \mathrm{GHz}$. To have finite conductivity in copper walls the insertion loss in each passband is maintained about $0.1 \mathrm{~dB}$. In Fig 2, the E-field norm is visualized for each pass band. The figure represents the input power at each passband is not split into two output ports, they are distributed independently without being coupled to the other port.

The E-field norm is visualized at the last frequency, which is out of the pass band. The plot shows that the input wave does not propagate to any of the output ports. Fig .3 shows the S-parameter plot of the lower and upper pass band of the diplexer. The isolation properties between two output ports are obtained by exciting port 2 or 3 only.

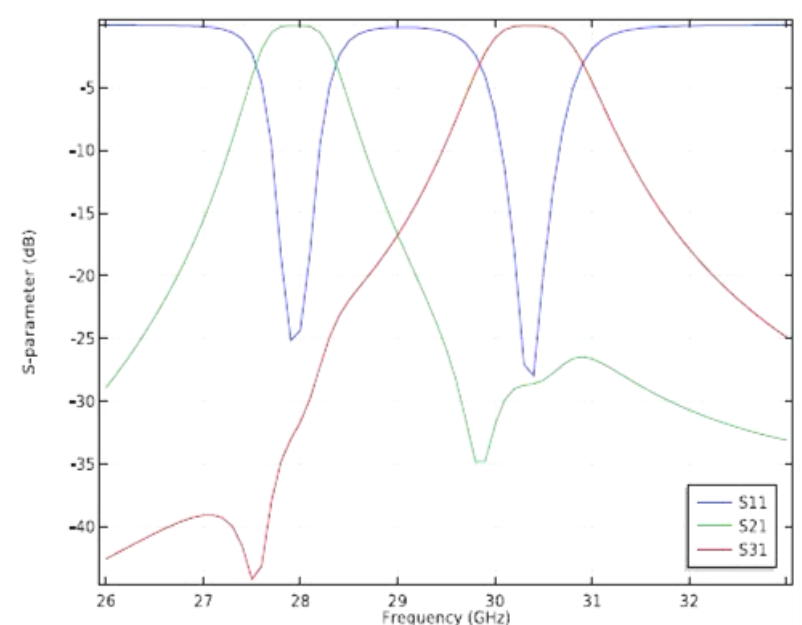

Fig. 3: S-Parameter Plot of the Lower and Upper Pass Band of the Diplexer

Table 2: Comparison Table of Various S Parameters with Respect to Its Operating Frequency (GHZ) of Diplexer.

\begin{tabular}{ll}
\hline S parameter $($ in $\mathrm{dB})$ & Operating frequency $(\mathrm{GHz})$ \\
\hline S11 & $28-30.4$ passband \\
S21 & $27.6-28.2$ passband \\
S31 & $30.2-30.8$ passband \\
\hline
\end{tabular}

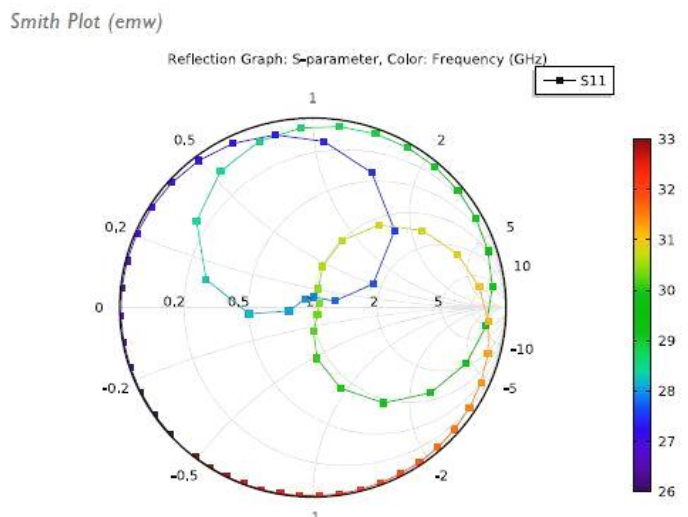

Fig. 4: Smith Plot (Emw)-Reflection Graph for S-Parameters (S11) of 2 Ports in Waveguides in Ghz Frequency.

From Fig 4 the result analysis for lower and upper pass band is obtained from the model window builder. The smith Plot shows the port outputs at $\mathrm{S} 11$ by selecting parameter value (freq $(\mathrm{GHz})$ ) with respect to Electric field (emw)

\section{Conclusion}

The arrival of $5 \mathrm{G}$ networks ensures that we are progressing towards reliable and faster wireless network compatible to all end users. The proposed work addresses the Waveguide diplexer can foster the high efficient network frequency spectrum in IoT devices. The Antenna magus software tool helps to accelerate the diplexer design and modeling process. The best $\mathrm{S}$ parameter configuration is examined at S11 with a frequency range of 28 to $30.4 \mathrm{GHz}$. In future, we 
can increase the Frequency spectrum using Phase progression technology [8] to use in various applications at wide range of frequencies.

\section{References}

[1] Wenlin Xia, Xiaobang Shang, Michael J. Lancaster: All-resonatorbased waveguide diplexer with cross- couplings. In: Electronics Letters,vol-50, ,pp 1948 - 195(2014).

[2] Fei Cheng; Xianqi Lin; Kaijun Song; Yuan Jiang; Yong Fan: Compact Diplexer With High Isolation Using the Dual-Mode Substrate Integrated Waveguide Resonator. In: IEEE Microwave and Wireless Components Letters. Vol- 23, pp̄: 459 -461(2013).

[3] F. Arndt, J. Bornemann, D. Grauerholz, D. Fasold, N. Schroeder, Waveguide E-plane integrated-circuit diplexer .In: Electronics Letters, Vol: 21,pp: 615 - 617 (1985).

[4] Luca Di Palma, Filiberto Bilotti, Alessandro Toscano, Lucio Vegni. Design of a Waveguide Diplexer Based on Connected Bi-Omega Particles. In: IEEE Microwave and Wireless Components Letters, Vol: 22, pp: 126- 128(2012).

[5] Hee-Ran Ahn, Manos M. Tentzeris. : Comments on On-Chip Miniaturized Diplexer Using Joint Dual-Mode Right-/Left-Handed Synthesized Coplanar Waveguides on GIPD Process. In: IEEE Microwave and Wireless Components Letters: Vol: 26 , pp: 380- 382 (2016).

[6] Tianning Zheng, Bin Wei; Bisong Cao, Xubo Guo, Xiaoping Zhang, Linan Jiang, Zhan Xu, Yong Heng .: Compact Superconducting Diplexer Design With Conductor-Backed Coplanar Waveguide Structures. In: IEEE Transactions on Applied Superconductivity. Vol: 25(2015).

[7] Z. Kordiboroujeni, J. Bornemann: K-band backward diplexer in substrate integrated waveguide technology. In: Electronics Letters, Year: 2015, Vol: 51.pp: 1428 -1429 (2015).

[8] Esperanza Alfonso Alós, Ashraf Uz Zaman, Per-Simon Kildal.:KaBand Gap Waveguide Coupled-Resonator Filter for Radio Link Diplexer Application. In: IEEE Transactions on Components, Packaging and Manufactüring Technology. Vol: 3, pp: 870- 879(2013). 